\title{
Effects of Sevelamer Hydrochloride on Uremic Toxins Serum Indoxyl Sulfate and P-Cresyl Sulfate in Hemodialysis Patients
}

\author{
Cheng-Jui Lin ${ }^{\mathrm{a}, \mathrm{b}, \mathrm{c}}$, Chi-Feng Pan ${ }^{\mathrm{a}, \mathrm{b}}$, Chih-Kuang Chuang, d, e, Hsuan-Liang Liu ${ }^{\mathrm{c}}$, \\ Sung-Fa Huang ${ }^{\mathrm{f}}$, Han-Hsiang Chen ${ }^{\mathrm{a}, \mathrm{b}}$, Chih-Jen $\mathrm{Wu}^{\mathrm{a}}$, b, g, h
}

\begin{abstract}
Background: Beside the phosphate binding effect, non-calcium nonaluminum phosphate binder, namely sevelamer hydrochloride (SH), has many other effects in dialysis patients. It can absorb many other compounds, decrease low-density lipoprotein cholesterol (LDL-C) level, and attenuate the progression of vascular calcification; it has been reported to have anti-inflammatory effect. However, it is not clear whether it has any effect on uremic toxins, i.e. serum indoxyl sulfate (IS) and p-cresyl sulfate, (PCS) in hemodialysis (HD) patients. This study was carried out to appraise the effect of sevelamer on serum IS and PCS in HD patients.
\end{abstract}

Methods: Five adult HD patients from a single medical center were enrolled in this study; these patients were treated with $800 \mathrm{mg}$ of sevelamer thrice per day for 3 months; a series of biochemical parameters, serum IS and PCS were monitored concurrently.

Results: There was a significant reduction in the mean level of phosphate from $7.20 \pm 0.70 \mathrm{mg} / \mathrm{dL}$ (mean $\pm \mathrm{SD}$ ) before treatment to $5.40 \pm$ $0.50 \mathrm{mg} / \mathrm{dL}$ (mean $\pm \mathrm{SD}$ ) after treatment, total cholesterol from 151.00 $\pm 37.40 \mathrm{mg} / \mathrm{dL}$ (mean $\pm \mathrm{SD}$ ) before treatment to $119.20 \pm 29.40 \mathrm{mg} /$ $\mathrm{dL}($ mean $\pm \mathrm{SD}$ ) after treatment, and PCS from $31.30 \pm 10.60 \mathrm{mg} / \mathrm{L}$ $($ mean $\pm \mathrm{SD})$ before treatment to $19.70 \pm 10.50 \mathrm{mg} / \mathrm{L}($ mean $\pm \mathrm{SD})$

Manuscript submitted February 16, 2014, accepted March 13, 2014

aDivision of Nephrology, Department of Internal Medicine, Mackay Memorial Hospital, Taipei, Taiwan, Republic of China

${ }^{\mathrm{b}}$ Mackay Junior College of Medicine, Nursing, and Management, Taipei, Taiwan, Republic of China

'Institute of Biotechnology, National Taipei University of Technology, Taipei, Taiwan, Republic of China

dDivision of Genetics and Metabolism, Department of Medical Research, Mackay Memorial Hospital, Taipei, Taiwan, Republic of China

${ }^{e}$ College of Medicine, Fu-Jen Catholic University, Taipei County, Taiwan, Republic of China

fDepartment of Laboratory Medicine, Mackay Memorial Hospital, Taipei, Taiwan, Republic of China

gGraduate Institute of Medical Science, Taipei Medical University, Taipei, Taiwan, Republic of China

${ }^{\mathrm{h}}$ Corresponding Author: Chih-Jen Wu, Division of Nephrology, Department of Internal Medicine, Mackay Memorial, Hospital, 92 Chung San North Road, Section 2, Taipei 104, Taiwan, Republic of China.

Email: lincj@ms1.mmh.org.tw

doi: https://doi.org/10.14740/jocmr1803e after treatment. On the contrary, this treatment had no effect on IS.

Conclusion: A statistically significant reduction of serum phosphate and PCS in HD patients treated with SH suggests that beside the action of lowering serum phosphate, sevelamer may have an important role in the treatment of uremic syndrome by decreasing the uremic toxin.

Keywords: P-cresyl sulfate; Indoxyl sulfate; Sevelamer hydrochloride; Hemodialysis

\section{Introduction}

Uremic syndromes are considered to be associated with uremic toxins and are compounds that accumulate in patients with end-stage renal disease (ESRD) [1]. Various disorders in patients with ESRD are associated with uremic toxins, and through dialysis, it is difficult to remove some of these toxins. These toxic compounds are not only involved in the etiology of uremic syndrome, but also have the potential to cause cardiovascular disease and renal osteodystrophy [2]. Evidences indicated that uremic toxins, namely indoxyl sulfate (IS) or pcresyl sulfate (PCS), increase the risk of cardiovascular event or mortality in patients with chronic kidney disease (CKD). Reducing the amount of phosphorus absorbed from the intestinal tract is one of the keystones of hyperparathyroidism prevention and treatment in such patients [3,4]. Over the past 40 years, phosphate binders have been used extensively for this purpose in dialysis patients; however, protein-bound uremic toxins are difficult to remove by dialysis [2].

Sevelamer hydrochloride (SH) is widely used as a non-calcium and non-aluminum phosphate binder in dialysis patients; however, in addition to its phosphate binding effect, $\mathrm{SH}$ adsorbs various other compounds. Treatment with SH has helped to decrease serum low-density lipoprotein cholesterol (LDL-C) levels in dialysis patients [5]. This lipid lowering effect of sevelamer is possibly due to its binding to bile acids [6]. SH has been shown to better attenuate the progression of vascular calcification compared with calcium containing phosphate binders [7], but some studies have no effective decline in vascular calcification [8-10]. In addition, it has been reported to have an anti-inflammatory effect and lowered serum high-sensitivity $\mathrm{C}$-reactive protein and $\beta 2$-microglobulin levels in hemodialysis 
Table 1. Baseline Characteristics of the Patients Before and 3 Months After Sevelamer Treatment

\begin{tabular}{|c|c|c|c|}
\hline \multirow{2}{*}{ Variables } & \multicolumn{2}{|c|}{ HD patients $(n=5)$} & \multirow{2}{*}{ P-value } \\
\hline & Before treatment & After treatment & \\
\hline Age (years) & $46 \pm 6.3$ & & \\
\hline Man & $20 \%$ & & \\
\hline Time of dialysis (years) & $5.3 \pm 1.1$ & & \\
\hline $\mathrm{cGN}$ & $100 \%$ & & \\
\hline $\mathrm{Kt} / \mathrm{V}$ & $1.5 \pm 0.1$ & $1.6 \pm 0.1$ & NS \\
\hline $\mathrm{SBP}(\mathrm{mm} \mathrm{Hg})$ & $138.4 \pm 13.2$ & $135.2 \pm 12.9$ & NS \\
\hline DBP (mm Hg) & $88.6 \pm 6.9$ & $90 \pm 7.5$ & NS \\
\hline $\mathrm{Hb}(\mathrm{g} / \mathrm{L})$ & $11.4 \pm 2.1$ & $11.7 \pm 2.2$ & NS \\
\hline $\mathrm{Ht}(\%)$ & $34.1 \pm 6.2$ & $35.3 \pm 6.5$ & NS \\
\hline $\mathrm{CO}_{2}(\mathrm{mmol} / \mathrm{L})$ & $22.2 \pm 1.3$ & $21.9 \pm 1.4$ & NS \\
\hline Glucose (AC) & $86.0 \pm 8.2$ & $89 \pm 8.3$ & NS \\
\hline Albumin (g/dL) & $4.0 \pm 0.3$ & $4.1 \pm 0.3$ & NS \\
\hline ALK-P (IU/L) & $301.0 \pm 216.8$ & $275 \pm 139.6$ & NS \\
\hline GOT (IU/L) & $14.8 \pm 6.3$ & $14.2 \pm 6.1$ & NS \\
\hline GPT (IU/L) & $13.0 \pm 4.7$ & $13.6 \pm 4.3$ & NS \\
\hline T-chol (mg/dL) & $151.0 \pm 37.4$ & $119.2 \pm 29.4$ & 0.02 \\
\hline TG (mg/dL) & $110.4 \pm 48.0$ & $95 \pm 17.6$ & NS \\
\hline Uric acid (mg/dL) & $7.7 \pm 1.2$ & $7.5 \pm 1.3$ & NS \\
\hline BUN (mg/dL) & $86.0 \pm 25.1$ & $91.4 \pm 27.4$ & NS \\
\hline Creatinine $(\mathrm{mg} / \mathrm{dL})$ & $10.9 \pm 2.0$ & $10.7 \pm 2.1$ & NS \\
\hline $\mathrm{K}(\mathrm{mEq} / \mathrm{L})$ & $5.3 \pm 0.6$ & $5.5 \pm 0.8$ & NS \\
\hline $\mathrm{Na}(\mathrm{mEq} / \mathrm{L})$ & $138.6 \pm 1.5$ & $136.7 \pm 1.4$ & NS \\
\hline $\mathrm{P}(\mathrm{mg} / \mathrm{dL})$ & $7.2 \pm 0.7$ & $5.4 \pm 0.5$ & $<0.01$ \\
\hline $\mathrm{Ca}(\mathrm{mg} / \mathrm{dL})$ & $9.7 \pm 0.5$ & $9.1 \pm 0.4$ & 0.01 \\
\hline i-PTH (pg/mL) & $984.8 \pm 346.2$ & $839.5 \pm 295.1$ & NS \\
\hline IS (mg/L) & $35.4 \pm 8.5$ & $32.1 \pm 8.1$ & NS \\
\hline PCS (mg/L) & $31.3 \pm 10.6$ & $19.7 \pm 10.5$ & $<0.01$ \\
\hline
\end{tabular}

(HD) patients [11]. However, studies did not demonstrate the effect of sevelamer on protein-binding uremic toxins, namely IS or PCS and hence this study aimed to investigate whether sevelamer treatment lowers IS and PCS in HD patients.

\section{Materials and Methods}

\section{Study patients}

This study enrolled five stable HD patients with hyperphosphatemia from January 2011 in a single medical center. Patients with acute infection, cardiovascular events in the past 3 months, malignancy or those younger than 18 years were excluded from this study. These patients underwent 4-h maintenance dialysis thrice a week using a synthetic dialysis membrane (polysulfone or polyamide). Dialyzers were not reused. Dialysis efficiency was calculated according to the Kidney
Disease Outcomes Quality Initiative (KDOQI) guidelines, and single-pool Kt/V of urea nitrogen was calculated [12]. All patients were treated with $800 \mathrm{mg}$ of $\mathrm{SH}$ thrice a day for 3 months. Also we offered the calcitriol $1 \mathrm{mcq}$ injection each HD session in our patients with hyperparathyroidism. A series of biochemical parameters, IS and PCS were monitored concurrently. This study was performed in accordance with the principles of the Declaration of Helsinki and approved by the Ethics Committee of the Mackay Memorial Hospital. Informed consent was obtained from all study patients.

\section{Laboratory assessment}

All blood samples were obtained just before the dialysis procedure, and the following biochemical tests were performed after treatment: blood glucose, blood urea nitrogen (BUN), creatinine $(\mathrm{Cr})$, glutamic oxaloacetic transaminase (GOT), glutamic pyruvic transaminase (GPT), alkaline phosphatase (ALK-P), 
Table 2. The Relationship of Serum Phosphate, IS and PCS Levels Before and After Treatment Analyzed by Trend Analysis

\begin{tabular}{llll} 
& F & df & P-value \\
\hline IS & 1.64 & 1 & 0.271 \\
PCS & 28.91 & 1 & 0.021 \\
Phosphate & 53.55 & 1 & 0.001 \\
\hline
\end{tabular}

hemoglobin (Hb), hematocrit (Hct), T-cholesterol (T-chol), triglyceride $(\mathrm{TG})$, sodium $(\mathrm{Na})$, potassium $(\mathrm{K})$, calcium $(\mathrm{Ca})$, phosphate $(\mathrm{P})$, bicarbonate, intact-parathyroid hormone (iPTH), albumin, IS and PCS. Albumin level was determined by bromocresol green method. Blood samples of patients were taken immediately before the HD session (mid-session in a week). Biochemical tests, including PCS and IS were measured every month after starting SH treatment for 3 months.

Serum PCS and IS were analyzed with ultra-performance liquid chromatography (UPLC). In summary, serum samples were prepared and deproteinized by heat denaturation. UPLC (ACQUITY UPLC ${ }^{\circledR}$ ) was performed at room temperature using a BEH phenyl column $(2.1 \times 100 \mathrm{~mm})$ and a photodiode array (PDA) detector at $280 \mathrm{~nm}$. The buffers used were: (A) $10 \mathrm{mM} \mathrm{NH}_{4} \mathrm{H}_{2} \mathrm{PO}_{4}(\mathrm{pH}=4.0)$ and (B) $100 \%$ acetonitrile. The flow rate was $0.4 \mathrm{~mL} / \mathrm{min}$ with a 9 -min gradient cycling from $82.5 \% \mathrm{~A} / 17.5 \% \mathrm{~B}$ to $55 \% \mathrm{~A} / 45 \% \mathrm{~B}$. Under these conditions, both PCS and IS were eluted at 2.75 and $1.4 \mathrm{~min}$, respectively. Standard curves for PCS and IS were set at $0.5,1,2.5,5$, and $10 \mathrm{mg} / \mathrm{L}$; both were processed in the same manner as the serum samples, and they correlated with the serum samples with average $\mathrm{r}^{2}$ values of $0.999 \pm 0.001$. These samples were diluted if the IS or PCS concentration exceeded standard curve. Quantitative results were obtained and calculated in terms of their concentrations $(\mathrm{mg} / \mathrm{L})$. The sensitivity of this assay was 0.425 $\mathrm{mg} / \mathrm{L}$ for PCS and $0.225 \mathrm{mg} / \mathrm{L}$ for IS

\section{Statistical analysis}

The demographic data were expressed as the mean $\pm \mathrm{SD}$. The comparisons of biochemical parameters in patients before and after a 3-month SH treatment were analyzed by paired and unpaired $t$-tests. In addition, the relationship of serum phosphate, IS and PCS levels before and after treatment was examined by trend analysis. A P value less than 0.05 was considered statistically significant. All statistical analyses were conducted by using the SPSS ver. 17.0 software programs (SPSS, Chicago, IL).

\section{Results}

We did not observe any significant differences in baseline characteristics regarding hypertension, ALK-P, GOT, GPT, TG, uric acid, BUN, creatinine, K, Na, and IS in HD and SH treated patients (Table 1). However, the serum levels of phosphorus, calcium and PCS were decreased significantly $(<0.01,0.01$ and $<0.01$ ) in 3 months of sevelamer treated patients compared with the baseline. In addition, the study also showed that
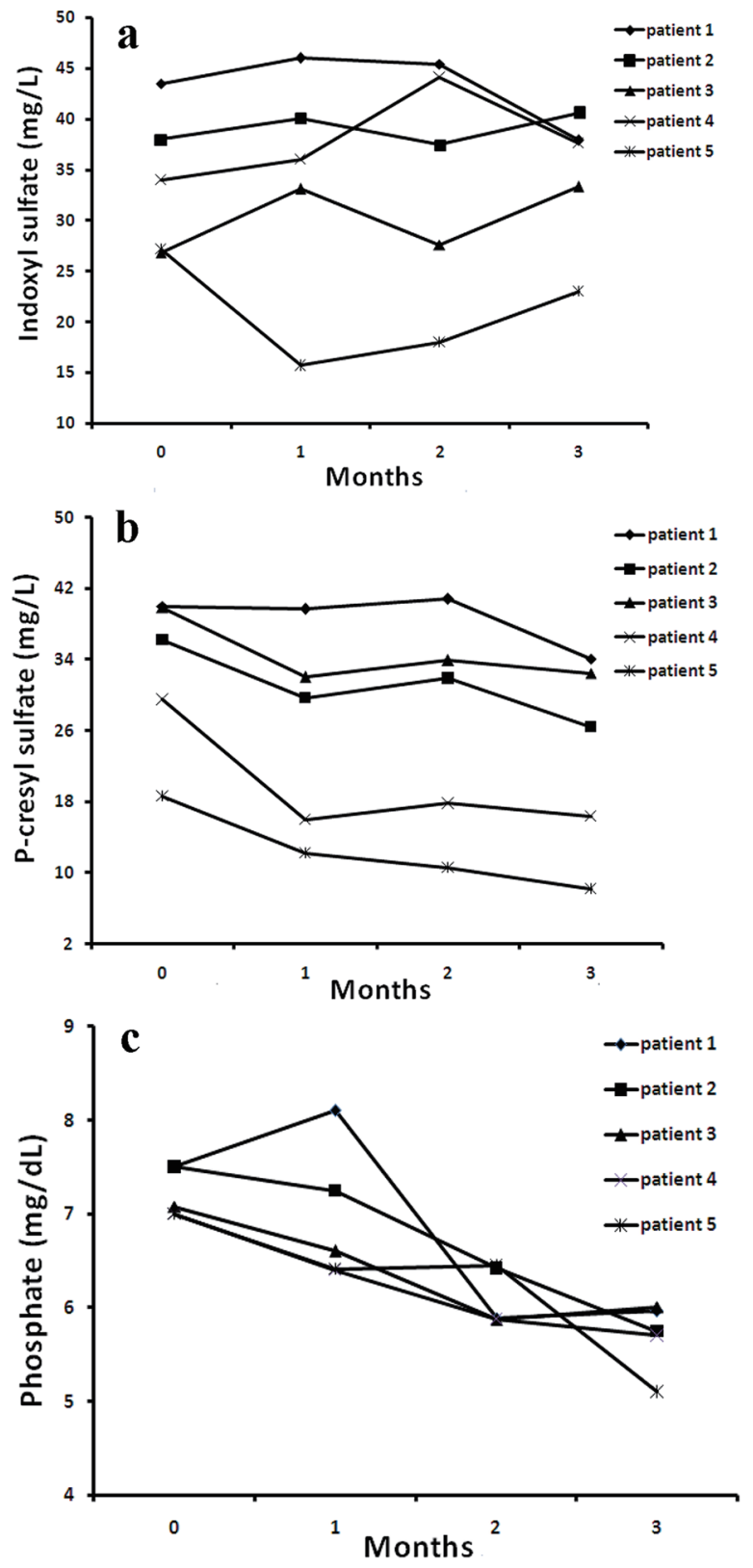

Figure 1. The distribution of serum IS (a), PCS (b) and phosphate (c) levels at 0, 1, 2, 3 months after SH treatment for all HD patients.

there was a significant decrease in the serum levels of T-chol in SH treated group $(151.0 \pm 37.4$ vs. $119 \pm 29.4, \mathrm{P}=0.02)$ (Table $1)$. With respect to the laboratory parameters at baseline, there was no much difference between the serum levels of IS (35.4 \pm $8.5 \mathrm{mg} / \mathrm{L})$ and PCS $(31.3 \pm 10.6 \mathrm{mg} / \mathrm{L})$.

Table 2 shows the trend analysis of serum levels of IS, PCS and phosphate before and after SH treatment in HD patients and the results revealed that phosphate and PCS were significantly reduced in HD patients who had been treated with $\mathrm{SH}$ for 3 months. However, this effect has not been described with the serum levels of IS (Table 2).

Figure 1 displays the serum levels of IS, PCS and phos- 

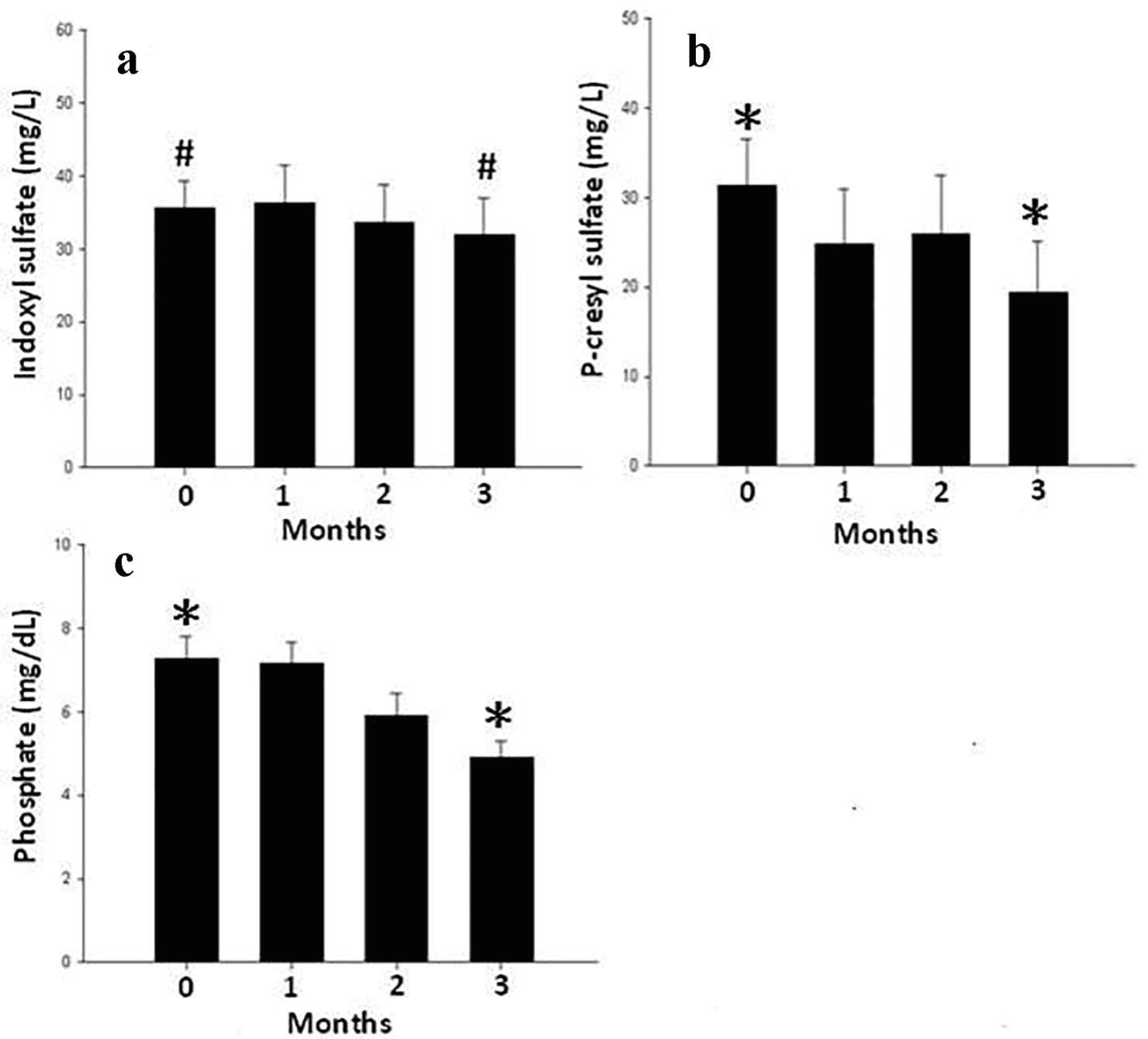

Figure 2. The average value of serum IS (a), PCS (b) and phosphate (c) levels at $0,1,2,3$ months after SH treatment for all HD patients. Data are mean \pm SEM. ${ }^{*} P<0.01, \# P=0.32$.

phate for $0,1,2$ and 3 months of sevelamer therapy in each of five HD patients. The results described that for HD patients who were treated with sevelamer, their serum concentrations of PCS and phosphate were significantly reduced. However, sevelamer therapy did not have effect on serum IS. In particular, among all patients, sevelamer therapy seemed to be more effective on patient number 5 .

These results were further confirmed as shown in Figure 2, where the average serum levels of PCS and phosphate were significantly reduced after 3 months of sevelamer treatment when compared to baseline. Moreover, this effect was not described on IS levels.

\section{Discussion}

Our study showed that the serum levels of phosphate, T-chol and PCS could be significantly decreased after 3 months of $\mathrm{SH}$ treatment in $\mathrm{HD}$ patients. It may suggest that $\mathrm{SH}$ had more specific binding for PCS than IS.

The treatment of hyperphosphatemia in ESRD has typically focused on the use of oral calcium-based phosphate binders taken at mealtimes, which bind dietary phosphate. Dietary phosphate binders can be used by virtually all ESRD patients. Calcium-based agents have traditionally been used as first line therapy, since they correct hypocalcemia in addition to reduce serum phosphate levels, and are inexpensive [13]. Agents such as non-calcium, non-magnesium and aluminum-free have newly become available for use as phosphate binders in patients with ESRD, of which sevelamer is the first such agent to be approved for use in North America [14]. Given the large differential cost between this new agent and traditional therapies, the optimal use of this new therapy requires consideration.

In the present observational study, five HD patients received sevelamer after calcium-based phosphate binders failed, and were evaluated for the effect of this drug on phosphate and PCS. As expected, this drug was effective in decreasing the serum levels of phosphate and PCS when compared to normal 
untreated HD patients. This favorable effect is independent of some classically established risk factors such as age, blood pressure, time of $\mathrm{HD}$ and $\mathrm{K}$ and dependent on $\mathrm{Ca}$, T-chol and P. However, we did not observe any significant effects of sevelamer on serum IS levels in HD patients.

The main mechanism of action of sevelamer is entrapment of endoluminal phosphorus in the gastrointestinal tract into non-absorbable complexes, which are excreted in feces. In addition to its primary indication as a phosphate binder, sevelamer also acts as a bile acid sequestrant in the gastrointestinal tract [6]. This is a potentially important activity, as CKD is associated with profound changes in lipid metabolism and serum lipid profiles [15]. Administration of sevelamer is associated with significant changes in the serum lipid profile of dialysis patients. Several prospective studies have consistently shown that total cholesterol and LDL-C levels decrease significantly with sevelamer treatment in dialysis patients [16-18]. Sevelamer is reported to cause a minimal decrease in the serum calcium concentration and LDL-C both in dialysis and predialysis patients [19]. Calcification is thought to result, at least in part, from the absorption and retention of calcium, and leads to arterial stiffening, which may result in cardiovascular events and death $[20,21]$. These results are highly corroborated findings from our study as it was found that total cholesterol and calcium significantly decreased after 3 months of sevelamer treatment.

IS and PCS were generated from colonic fermentation product and linked to cardiovascular disease and mortality in dialysis patients [22-24]. Serum levels of IS was reported to be associated with dialysis vintage [25]. It is therefore tempting to speculate that strictly endoluminally acting substances, such as sevelamer, could modify the composition of distal bowel content, influence colonic fermentation, and consequently, alter the generation and absorption of such uremic toxins. In addition, the effect of $\mathrm{SH}$ on protein-binding uremic toxins is unclear. An in vitro study showed that $\mathrm{SH}$ binds indole (10$15 \%)$ and p-cresol (40-50\%) [26]. Phan et al investigated the effect of SH on serum IS levels in 10 chronic HD patients with uncontrolled hyperphosphatemia and found that it significantly decreased serum phosphate levels and increased IS levels [27]. One recent report suggested that sevelamer was effective in lowering p-cresol levels in a peritoneal dialysis cohort [28]. In this study, we also observed the effect of $\mathrm{SH}$ on lowering serum PCS, but not in IS, which may be possibly due to its high and low binding efficacy with p-cresol and indole, respectively. These findings are harmonized and supported the results derived from this study.

A recent study has also described that oligofructose enriched inulin decreased p-cresol generation, resulting in a reduction of serum concentrations of PCS by on average $20 \%$, and they also found that serum concentration of IS was not reduced by oligofructose enriched inulin treatment [29]. Our results are highly consistent with these data, in which $\mathrm{SH}$ is not effective on IS. However, there are limitations in our study. First, this study was performed with only minimum numbers of patients, and all subjects were enrolled from one medical center. Second, the definite mechanism of how does $\mathrm{SH}$ absorb PCS not IS is unclear. Further more studies are needed to resolve these questions.

\section{Conclusion}

Even though the elimination of uremic toxins in patients with CKD is essential, their removal by dialysis treatment is not easy and not absolutely flourishing. Uremic toxins can be decreased by oral adsorbents in the body when it binds with them and have the potential to provide clinical benefits. Various compounds related to uremic toxins are absorbed by AST-120, and it is thus believed that it may recover disorders related to CKD. SH hampers with the absorption of other dangerous compounds and thus may tender additional benefits, other than its action of lowering serum phosphate. It thus suggests that sevelamer therapy is an important modality in the treatment of uremic syndrome.

\section{Acknowledgments}

We would like to thank all the patients who were involved in this study.

\section{Grant}

This study was supported in part by a grant from National Science Council of Taiwan (NSC 100-2314-B-195-012).

\section{Conflicts of Interest}

No relevant conflicts of interest.

\section{References}

1. Vanholder R, De Smet R, Glorieux G, Argiles A, Baurmeister U, Brunet P, Clark W, et al. Review on uremic toxins: classification, concentration, and interindividual variability. Kidney Int. 2003;63(5):1934-1943.

2. Coladonato JA. Control of hyperphosphatemia among patients with ESRD. J Am Soc Nephrol. 2005;16(Suppl 2):S107-114.

3. Slatopolsky E. New developments in hyperphosphatemia management. J Am Soc Nephrol. 2003;14(9 Suppl 4):S297299.

4. Vanholder R, Baurmeister U, Brunet P, Cohen G, Glorieux G, Jankowski J, European Uremic Toxin Work G. A bench to bedside view of uremic toxins. J Am Soc Nephrol. 2008;19(5):863-870.

5. Wilkes BM, Reiner D, Kern M, Burke S. Simultaneous lowering of serum phosphate and LDL-cholesterol by sevelamer hydrochloride (RenaGel) in dialysis patients. Clin Nephrol. 1998;50(6):381-386.

6. Braunlin W, Zhorov E, Guo A, Apruzzese W, Xu Q, Hook $\mathrm{P}$, Smisek DL, et al. Bile acid binding to sevelamer $\mathrm{HCl}$. Kidney Int. 2002;62(2):611-619.

7. Chertow GM, Raggi P, Chasan-Taber S, Bommer J, Holzer H, Burke SK. Determinants of progressive vascular calcification in haemodialysis patients. Nephrol Dial 
Transplant. 2004;19(6):1489-1496.

8. Block GA, Wheeler DC, Persky MS, Kestenbaum B, Ketteler M, Spiegel DM, Allison MA, et al. Effects of phosphate binders in moderate CKD. J Am Soc Nephrol. 2012;23(8):1407-1415.

9. Qunibi W, Moustafa M, Muenz LR, He DY, Kessler PD, Diaz-Buxo JA, Budoff M, et al. A 1-year randomized trial of calcium acetate versus sevelamer on progression of coronary artery calcification in hemodialysis patients with comparable lipid control: the Calcium Acetate Renagel Evaluation-2 (CARE-2) study. Am J Kidney Dis. 2008;51(6):952-965.

10. Qunibi WY, Hootkins RE, McDowell LL, Meyer MS, Simon M, Garza RO, Pelham RW, et al. Treatment of hyperphosphatemia in hemodialysis patients: The Calcium Acetate Renagel Evaluation (CARE Study). Kidney Int. 2004;65(5):1914-1926.

11. Ferramosca E, Burke S, Chasan-Taber S, Ratti C, Chertow GM, Raggi P. Potential antiatherogenic and anti-inflammatory properties of sevelamer in maintenance hemodialysis patients. Am Heart J. 2005;149(5):820-825.

12. Daugirdas JT. The post: pre-dialysis plasma urea nitrogen ratio to estimate K.t/V and NPCR: mathematical modeling. Int J Artif Organs. 1989;12(7):411-419.

13. Manns B, Stevens L, Miskulin D, Owen WF, Jr., Winkelmayer WC, Tonelli M. A systematic review of sevelamer in ESRD and an analysis of its potential economic impact in Canada and the United States. Kidney Int. 2004;66(3):1239-1247.

14. Jamal SA, Vandermeer B, Raggi P, Mendelssohn DC, Chatterley T, Dorgan M, Lok CE, et al. Effect of calcium-based versus non-calcium-based phosphate binders on mortality in patients with chronic kidney disease: an updated systematic review and meta-analysis. Lancet. 2013;382(9900):1268-1277.

15. Trevisan R, Dodesini AR, Lepore G. Lipids and renal disease. J Am Soc Nephrol. 2006;17(4 Suppl 2):S145-147.

16. Chertow GM, Burke SK, Raggi P, Treat to Goal Working G. Sevelamer attenuates the progression of coronary and aortic calcification in hemodialysis patients. Kidney Int. 2002;62(1):245-252.

17. Brandenburg VM, Jahnen-Dechent W, Ketteler M. Sevelamer and the bone-vascular axis in chronic kidney disease: bone turnover, inflammation, and calcification regulation. Kidney Int Suppl. 2009;114:S26-33.

18. Goldberg DI, Dillon MA, Slatopolsky EA, Garrett B, Gray JR, Marbury T, Weinberg M, et al. Effect of RenaGel, a non-absorbed, calcium- and aluminium-free phosphate binder, on serum phosphorus, calcium, and intact parathyroid hormone in end-stage renal disease patients. Nephrol Dial Transplant. 1998;13(9):2303-2310.

19. Ketteler M, Rix M, Fan S, Pritchard N, Oestergaard O, Chasan-Taber S, Heaton J, et al. Efficacy and tolerability of sevelamer carbonate in hyperphosphatemic patients who have chronic kidney disease and are not on dialysis. Clin J Am Soc Nephrol. 2008;3(4):1125-1130.

20. Guerin AP, London GM, Marchais SJ, Metivier F. Arterial stiffening and vascular calcifications in end-stage renal disease. Nephrol Dial Transplant. 2000;15(7):1014-1021.

21. Blacher J, Guerin AP, Pannier B, Marchais SJ, Safar ME, London GM. Impact of aortic stiffness on survival in endstage renal disease. Circulation. 1999;99(18):2434-2439.

22. Meijers BK, Bammens B, De Moor B, Verbeke K, Vanrenterghem Y, Evenepoel P. Free p-cresol is associated with cardiovascular disease in hemodialysis patients. Kidney Int. 2008;73(10):1174-1180.

23. Lin CJ, Wu CJ, Pan CF, Chen YC, Sun FJ, Chen HH. Serum protein-bound uraemic toxins and clinical outcomes in haemodialysis patients. Nephrol Dial Transplant. 2010;25(11):3693-3700.

24. Bammens B, Evenepoel P, Keuleers H, Verbeke K, Vanrenterghem Y. Free serum concentrations of the proteinbound retention solute p-cresol predict mortality in hemodialysis patients. Kidney Int. 2006;69(6):1081-1087.

25. Lin CJ, Chuang CK, Liu HL, Wang TJ, Chen HH, Sun FJ, Wu CJ. The serum levels of P-cresol and Indoxyl sulfate in different hemodialysis vintage. J Clin Med Res. 2011;3(8):114-119.

26. De Smet R, Thermote F, Lamiere N. Sevelamer hydrochloride adsorbs the uremic compound indoxyl sulfate [abstract]. J Am Soc Nephrol. 2003;14:206A.

27. Phan O, Ivanovski O, Nguyen-Khoa T, Mothu N, Angulo J, Westenfeld R, Ketteler M, et al. Sevelamer prevents uremia-enhanced atherosclerosis progression in apolipoprotein E-deficient mice. Circulation. 2005;112(18):28752882.

28. Guida B, Cataldi M, Riccio E, Grumetto L, Pota A, Borrelli S, Memoli A, et al. Plasma p-cresol lowering effect of sevelamer in peritoneal dialysis patients: evidence from a Cross-Sectional Observational Study. PLoS One. 2013;8(8):e73558.

29. Meijers BK, De Preter V, Verbeke K, Vanrenterghem Y, Evenepoel P. p-Cresyl sulfate serum concentrations in haemodialysis patients are reduced by the prebiotic oligofructose-enriched inulin. Nephrol Dial Transplant. 2010;25(1):219-224. 